\title{
DISTRIBUIÇÃO POR ESTADO FÍSICO ASA E POR ESCALA DE MALLAMPATI DE PACIENTES SUBMETIDOS À HERNIORRAFIAS INGUINAIS POR VIDEOLAPAROSCOPIA
}

\section{ARTIGO ORIGINAL}

BEDIN, Rafael Antonio Caldart ${ }^{1}$

SCHULTZ, Maísa ${ }^{2}$

GUIMARÃES, André Wanderley de Oliveira ${ }^{3}$

KÜLKAMP, Felippe Orli de Farias Motta ${ }^{4}$

BEDIN, Antonio ${ }^{5}$

BEDIN, Rafael Antonio Caldart. Et al. Distribuição por estado físico ASA e por Escala de Mallampati de pacientes submetidos à herniorrafias inguinais por videolaparoscopia. Revista Científica Multidisciplinar Núcleo do Conhecimento. Ano 05, Ed. 10, Vol. 13, pp. 90-99. Outubro de 2020. ISSN: 2448-0959, Link de acesso: https://www.nucleodoconhecimento.com.br/saude/escala-de-mallampati

\section{RESUMO}

$\mathrm{Na}$ avaliação pré-anestésica, que deve sempre ocorrer antes de cirurgias eletivas, o anestesista realiza a avaliação do estado físico. Neste estudo verificou-se o perfil dos pacientes encaminhados para cirurgia de herniorrafia inguinal em Joinville segundo os critérios da Associação Americana de Anestesiologia no período de agosto de 2016

\footnotetext{
${ }^{1}$ Graduando de medicina.

${ }^{2}$ Graduanda de medicina.

${ }^{3}$ Graduando de medicina.

${ }^{4}$ Graduando de medicina.

${ }^{5}$ Orientador. Médico Anestesiologista. Mestre em Saúde. Doutor em Anestesiologia.
} 
a agosto de 2017. Avaliou-se o estado físico de acordo com a classificação da Associação Americana de Anestesiologia (ASA), identificando a classificação de estado físico (ASA 1, 2, 3, 4, 5 e 6) dos pacientes submetidos a cirurgia de herniorrafia inguinal em Joinville. Verificou-se a prevalência de via aérea 1, 2, 3 e 4 segundo escala de Mallampati. E correlacionou os dados para verificação e elaboração de abordagens terapêuticas individualizadas conforme os perfis encontrados para a melhoria do atendimento a pacientes. Este foi um estudo clínico epidemiológico retrospectivo realizado no período de agosto de 2016 a Agosto de 2017. 328 pacientes participaram do estudo sendo idade $45,80 \pm 11,60$, peso $81,22 \pm 13,32$ e índice de massa corporal 27,28 $\pm 3,79$. O estado físico, conforme a classificação da American Society o Anesthesiology (ASA), mais frequente foi ASA I. Escala de previsão de dificuldade de intubação traqueal Mallampati mais frequente foi 1. Este estudo teve como finalidade quantificar a prevalência do estado físico ASA e da classificação de Mallampati dos pacientes submetidos a cirurgia de herniorrafia inguinal na cidade de Joinville entre Agosto de 2016 e Agosto de 2017. Além disso, foram coletados dados demográficos (sexo, idade, peso e índice de massa corporal) como também estado físico e a classificação de previsão de dificuldade de intubação de Mallampati.

Palavras-chave: Anestesia geral, Herniorrafia, intubação, cuidados pré-operatórios.

\section{INTRODUÇÃO}

Na avaliação pré-anestésica, que deve sempre ocorrer antes de cirurgias eletivas, é função do anestesista realizar a avaliação do estado físico (CARVALHO e colab., 2019); (GREEN e ROBACK, 2019). Essa avaliação é feita segundo os moldes da American Society of Anesthesiologists (ASA) é um importante dado, de modo que o médico anestesiologista tenha a dimensão do potencial de risco durante o procedimento anestésico (MCISAAC e colab., 2017). Também é realizada a avaliação de viabilidade das vias aéreas, que pode ser feita segundo a escala de Mallampati, que é o preditor de dificuldade de via aérea mais utilizado pela anestesiologia atualmente(TELICH-TARRIBA e colab., 2019). 
Diante do uso em muitos serviços, sendo preditores de diversas situações clínicas e pós-operatórias(MADDALI e colab., 2018), a classificação de estado físico ASA e a classificação de Mallampati de perviedade das vias aéreas podem providenciar interessantes dados estatísticos para estudo (CARVALHO e colab., 2019).

Como citado em Mathias (1997) e em Fernandas Mendes et al., (2011),"O propósito principal da avaliação pré-operatória é reduzir a morbimortalidade do paciente cirúrgico.", assim desde Vacanti et al. (S.d.), sabe-se que há estreita relação entre a classificação de estado físico ASA e mortalidade em cirurgia. Tantos outros autores, como Davenport et al. (2006), Schwartzman et al. (2014 e 2011), Santos et al. (2017) e Wolters et al. (1996), fizeram a relação entre a avaliação pré-anestésica e o estado físico com complicações em cirurgia e anestesia, o que coloca esses fatores como objetos importantes de estudo para uma melhor prática médica (FUJIWARA et al., 2019).

\section{HIPÓTESE}

A prevalência dos fatores avaliados foi o esperado conforme a literatura.

\section{MÉTODOS}

O estudo foi iniciado após a aprovação pelo comitê de Ética em Pesquisa da Univille. Tratou-se de um estudo clínico epidemiológico prospectivo a ser realizado na Clínica de Anestesiologia na cidade de Joinville de agosto de 2016 a agosto de 2017.

\section{INCLUSÃO}

Pacientes que foram submetidos à cirurgia de herniorrafia inguinal e que tiveram sua avaliação pré-anestésica na Clínica de Avaliação Pré-anestésica do Serviço de Anestesiologia de Joinville de agosto de 2016 a agosto de 2017.

\section{EXCLUSÃO}

Pacientes estado físico ASA V e VI. 


\section{ESTATÍSTICA}

Para a análise de dados foi utilizada estatística descritiva (média, moda, mediana e desvio padrão) e utilizado o programa Microsoft Excel ${ }^{\circledR}$

\section{RESULTADOS}

Este foi um estudo clínico epidemiológico retrospectivo realizado na clínica de avaliação pré-anestésica do Serviço de Anestesiologia de Joinville na cidade de Joinville, Estado de Santa Catarina, Brasil, no período de agosto de 2016 a agosto de 2017. Foi aprovado pelo Comitê de Ética da Univille sob o número 3.249.237 (anexo). Foram analisados dados demográficos tais como idade, peso em quilos e índice de massa corporal (tabela 1). O estado físico segundo a classificação da American Society o Anesthesiology (ASA), (Tabela 2). Escala de previsão de dificuldade de intubação traqueal Mallampati (tabela 3). A análise estatística foi realizada pelo software Microsoft Excel $365^{\circledR}$.

Tabela 1. Dados demográficos expressos em média \pm desvio padrão.

\begin{tabular}{|l|l|}
\hline Número total $(\mathbf{n})$ & 328 \\
\hline Sexo (M/F) & $211 / 117$ \\
\hline Idade (média \pm desvio padrão) & $45,80 \pm 11,60$ \\
\hline Peso em quilos (média \pm desvio padrão) & $81,22 \pm 13,32$ \\
\hline IMC (média \pm desvio padrão) & $27,28 \pm 3,79$ \\
\hline
\end{tabular}

Fonte: autor.

Tabela 2. Estado físico ASA.

\begin{tabular}{|l|l|l|}
\hline Estado físico & Número & Porcentagem \\
\hline ASA I & $\mathbf{1 8 2}$ & $\mathbf{5 5 , 4 8}$ \\
\hline ASA II & $\mathbf{1 0 3}$ & $\mathbf{3 1 , 4 0}$ \\
\hline ASA III & $\mathbf{4 3}$ & $\mathbf{1 3 , 1 0}$ \\
\hline
\end{tabular}




\begin{tabular}{|l|l|l|}
\hline ASA IV & 0 & 0 \\
\hline ASA V & 0 & 0 \\
\hline AS VI & $\mathbf{0}$ & 0 \\
\hline
\end{tabular}

Fonte: autor.

Tabela 3. Escala de Mallampati

\begin{tabular}{|l|l|l|}
\hline Escala de Mallampati & Número & Porcentagem \\
\hline MLP 1 & $\mathbf{2 2 9}$ & $\mathbf{6 9 , 8 1}$ \\
\hline MLP 2 & $\mathbf{8 2}$ & $\mathbf{2 5 , 0 0}$ \\
\hline MLP 3 & $\mathbf{1 3}$ & $\mathbf{3 , 9 6}$ \\
\hline MLP 4 & $\mathbf{4}$ & $\mathbf{1 , 2 1}$ \\
\hline
\end{tabular}

Fonte: autor.

\section{DISCUSSÃO}

Vacanti et. al. (S.d.), realizaram uma extensa análise estatística sobre a relação entre estado físico e mortalidade dos pacientes no pós-operatório (TUNCALI et al., 2018). Sendo assim, Schwartzman et al. (2014) e Santos et al. (2017), discutiram sobre a importância da avaliação pré-anestésica no prognóstico e na possibilidade de intercorrências em cirurgias. De acordo com os autores, os pacientes mais debilitados em seu estado físico (ASA 3 e 4) são mais propensos a sofrerem complicações relacionadas à anestesia. Segundo o estudo, as chances da ocorrência de uma complicação relacionada à anestesia, está relativamente ligada a condição do estado físico do paciente, que ocorreram do seguinte modo: 20,4\% dos pacientes classificados como ASA apresentaram complicações I; entre aqueles julgados ASA II, $22,3 \%$ dos pacientes sofreram de complicações; $42,6 \%$ dos pacientes ASA III tiveram alguma complicação e, de maneira mais acentuada, complicações foram observadas em $100 \%$ dos pacientes ASA IV, tornando esses pacientes estatisticamente 16,7 vezes mais propensos de apresentar complicações relacionadas à anestesia em comparação com o pacientes ASA I(FUJIWARA e colab., 2019). 
No estudo realizado por Wolters et al. (1996) dados sobre a incidência dos estados ASA foram levantados correlacionando-os com os aspectos intra e pós-operatórios. Neste estudo, $18 \%$ dos pacientes foram classificados como ASA I, enquanto $42,6 \%$ como ASA II, 34,6\% como ASA III, 4,6\% como ASA IV e $0,2 \%$ como ASA V.

Nesse mesmo estudo foi verificado que entre os pacientes ASA I e os pacientes ASA III e IV ocorreu um aumento no tempo de duração da cirurgia, bem como entre os pacientes ASA II e ASA III. A perda de sangue também foi um aspecto relacionado com o estado ASA, demonstrando que pacientes ASA IV perdem mais sangue que os pacientes de ASA I, ASA II e ASA III. Outro ponto observado foi o tempo de ventilação pós-operatória o qual evidenciou um aumento de 2 a 6 vezes entre os grupos $(1,1 \mathrm{~h}$ para ASA I, 4,2h para ASA II, 7,7h para ASA III e 45,5h para ASA IV). Assim, o estado ASA influência de forma direta, o tempo de estadia no hospital, submetendo o paciente a um cuidado intensivo, em que pacientes com estados ASA mais baixos precisam ficar menos tempo no hospital se comparado a pacientes com estado ASA mais alto. Quanto as complicações de acordo com os níveis ASA, observou-se um aumento de 2 a 3 vezes nas complicações broncopulmonares, ao mesmo tempo que nas cardíacas foi aferido um aumento de 3 vezes. Por fim, foi verificada uma mortalidade de $0,1 \%$ para estados ASA I, 0,7\% para ASA II, 3,5\% para ASA III, 18,3\% para ASA IV e $93,3 \%$ para ASA V.

Outro fator importante da avaliação pré-operatória (dentro da parte de avaliação física), de acordo com Mathias (1997)(SOUZA e colab., 2005), é a avaliação da perviedade das vias aéreas, que tem como objetivo predizer a dificuldade de intubação do paciente. Um modo de realizar essa avaliação é com a classificação de Mallampati, que é frequentemente usada, como visto em Lundstrøm et al. (2011)(LUNDSTRØM e colab., 2011) e Shiga et al. (2005)(SHIGA e colab., 2005).

Diante do uso em muitos serviços, sendo preditores de diversas situações clínicas e pós-operatórias, a classificação de estado físico ASA e a classificação de Mallampati de perviedade das vias aéreas podem providenciar interessantes dados estatísticos para estudo. A classificação de estado físico ASA, além de estar relacionada à predição de complicações em anestesia segundo o estudo de Schwartzman et al. 
(2014) e Shiga et al. (2005), assim como a classificação de Mallampati em Mathias (1997)(FERNANDAS MENDES e colab., 2011), por definição está também relacionada à existência de doenças sistêmicas.

\section{CONCLUSÃO}

O presente estudo teve como objetivo quantificar a prevalência do estado físico ASA e da classificação de Mallampati dos pacientes submetidos a cirurgia de herniorrafia inguinal na cidade de Joinville entre agosto de 2016 e agosto de 2017. Além disso, foram coletados dados demográficos (sexo, idade, peso e índice de massa corporal) como também estado físico e à classificação de previsão de dificuldade de intubação de Mallampati. Dito isso, vale salientar que esses são temas pouco estudados pela população e, dada a clara importância de ambas estas classificações quanto a desfechos anestésicos e, consequentemente, cirúrgicos, este estudo epidemiológico demonstrou que estas variáveis "estado físico ASA" e "classificação de Mallampati" podem auxiliar no entendimento sobre tais variáveis na nossa população, o que poderá suscitar novos estudos nesta área sendo de grande valia para a anestesiologia, que é provável para o início de um procedimento cirúrgico necessariamente realizado sob anestesia geral e ventilação controlada mecânica, como são as herniorrafias inguinais por videolaparoscopia.

\section{REFERÊNCIAS}

CARVALHO, C. C. e colab. Pre-operative voice evaluation as a hypothetical predictor of difficult laryngoscopy. Anaesthesia, v. 74, n. 9, p. 1147-1152, 11 Set 2019. Disponível em: <https://onlinelibrary.wiley.com/doi/abs/10.1111/anae.14732>. Acesso em: 29 set 2019.

DAVENPORT, D. L. e colab. National Surgical Quality Improvement Program (NSQIP) Risk Factors Can Be Used to Validate American Society of Anesthesiologists Physical Status Classification (ASA PS) Levels. Annals of Surgery, v. 243, n. 5, p. 636, Maio 2006. Disponível em: <http://www.ncbi.nlm.nih.gov/pubmed/16632998>. Acesso em: 28 set 2019. 
FERNANDAS MENDES, F. e colab. Impacto da implantação de clínica de avaliação pré-operatória em indicadores de desempenho. Revista Brasileira de Anestesiologia, v. 61, n. 6, p. 786-792, 2011.

FUJIWARA, K. e colab. Preoperative predictors of difficult hypopharyngeal exposure by retractor for transoral robotic surgery. International Journal of Clinical Oncology, v. 24, n. 1, p. 53-59, 13 Jan 2019. Disponível em: <http://www.ncbi.nlm.nih.gov/pubmed/30101389>. Acesso em: 29 set 2019.

GREEN, S. M. e ROBACK, Mark G. Is the Mallampati Score Useful for Emergency Department Airway Management or Procedural Sedation? Annals of Emergency Medicine, v. 74, n. 2, p. 251-259, Ago 2019. Disponível em: <http://www.ncbi.nlm.nih.gov/pubmed/30782435>. Acesso em: 29 set 2019.

LUNDSTRØM, L.H. e colab. Poor prognostic value of the modified Mallampati score: a meta-analysis involving 177088 patients. British Journal of Anaesthesia, v. $107, \quad$ n. 5 , p. 659-667, Nov 2011. Disponível em: <http://www.ncbi.nlm.nih.gov/pubmed/21948956>. Acesso em: 28 set 2019.

MADDALI, M. e colab. Preoperative predictors of poor laryngoscope views in pediatric population undergoing cardiac catheterization. Annals of Cardiac Anaesthesia, v. 21, n. 4, p. 376, 2018. Disponível em: <http://www.ncbi.nlm.nih.gov/pubmed/30333330>. Acesso em: 29 set 2019.

MCISAAC, D. I. e colab. Effect of Preoperative Geriatric Evaluation on Outcomes After Elective Surgery: A Population-Based Study. Journal of the American Geriatrics Society, v. 65, n. 12, p. 2665-2672, Dez 2017. Disponível em: <http://www.ncbi.nlm.nih.gov/pubmed/28971482>. Acesso em: 29 set 2019.

SANTOS, M. e NOVAES, C. e IGLESIAS, A. C. Epidemiological profile of patients seen in the pre-anesthetic assessment clinic of a university hospital. Brazilian Journal of Anesthesiology, v. 67, n. 5, p. 457-467, 2017. Disponível em: $<$ http://dx.doi.org/10.1016/j.bjan.2016.06.002>. 
SHIGA, T. e colab. Predicting Difficult Intubation in Apparently Normal Patients. Anesthesiology, v. 103, n. 2, p. 429-437, Ago 2005. Disponível em: <http://www.ncbi.nlm.nih.gov/pubmed/16052126>. Acesso em: 28 set 2019.

SOUZA, F. S. e colab. Validade da rotina de realização do eletrocardiograma na avaliação pré-operatória de idosos. Revista Brasileira de Anestesiologia, v. 55, n. 1, p. $\quad 59-71, \quad$ Fev $2005 . \quad$ Disponível em: <http://www.scielo.br/scielo.php?script=sci_arttext\&pid=S003470942005000100007\&lng=pt\&nrm=iso\&tIng=pt $>$. Acesso em: 28 set 2019.

TELICH-TARRIBA, J. E. e colab. Difficult Airway Identification and Management in Patients With Moebius Syndrome. Journal of Craniofacial Surgery, p. 1, 28 Jun 2019. Disponível em: <http://www.ncbi.nlm.nih.gov/pubmed/31261321>. Acesso em: 29 set 2019.

TUNCALI, B. e colab. Retrospective Evaluation of Patients who Underwent Laparoscopic Bariatric Surgery. Turkish Journal of Anesthesia and Reanimation, v. 46, n. 4, p. 297-304, 16 Ago 2018. Disponível em: <http://www.ncbi.nlm.nih.gov/pubmed/30140537>. Acesso em: 29 set 2019.

VACANTI, C J e VANHOUTEN, R J e HILL, R C. A statistical analysis of the relationship of physical status to postoperative mortality in 68,388 cases. Anesthesia and analgesia, v. 49, n. 4, p. 564-6, [S.d.]. Disponível em: <http://www.ncbi.nlm.nih.gov/pubmed/5534668>. Acesso em: 28 set 2019.

WOLTERS, $U$ e colab. ASA classification and perioperative variables as predictors of postoperative outcome. British Journal of Anaesthesia, v. 77, n. 2, p. 217-222, Ago 1996. Disponível em: <http://www.ncbi.nlm.nih.gov/pubmed/8881629>. Acesso em: 28 set 2019.

Enviado: Setembro, 2020.

Aprovado: Outubro, 2020. 